\title{
3 Die Rhetorik des Amateurs
}

\section{Dilettantismen und Vorzüge der ungeschliffenen Alltagsrede}

\subsection{Einführung}

Neben den bisher genannten Formen der Imperfektion, die mit strategischer Absicht, als rhetorische Maßnahme in besonderen Situationen oder gar als Zeichen höchster Redekunst in die Rede eingebaut werden, resultiert eine große Anzahl von Imperfektionen in der Rede ganz einfach aus der Nichtbeherrschung der Téchnē. Verstöße gegen die Tugenden der Rede, d.h. gegen Sprachrichtigkeit, Klarheit, Schmuck oder Angemessenheit, sind innerhalb des rhetorischen Systems jedoch nur dann erlaubt, wenn sie kunstvoll und fachgerecht, mit Überlegung und Bedacht eingesetzt werden. Geschieht eine Abweichung zufällig, unabsichtlich oder gar kontraintentional, ist dies aus Sicht der klassischen Rhetorik klar als Fehler zu verurteilen. In der ungewollt imperfekten Rede offenbaren sich die rhetorisch Unbewanderten und Ungebildeten, die Dilettanten, Amateure oder Laien. ${ }^{1}$ Das dilettantisch Imperfekte zeigt sich in jeder Redeform, welche

1 Die Bezeichnungen 〈Laie〉, 〈Amateur〉 und 〈Dilettant〉 (ähnlich auch: 〈Anfänger〉) sowie die entsprechenden Adjektive 〈laienhaft), ‘amateurhaft und «dilettantisch〉 werden in dieser Arbeit grundsätzlich synonym verwendet. Zwar stammen die Wörter aus unterschiedlichen historischen Kontexten, sind teilweise unterschiedlich konnotiert und werden nicht ganz deckungsgleich angewendet. So schwingt in 〈Amateur die Liebhaberei und in «Dilettant〉 die Freude (ital. dilettare - sich erfreuen) mit, was auf eine leidenschaftlichere Betreibung der entsprechenden Tätigkeit oder Kunst hindeutet als die Bezeichnung «Laie`. Dem Wort «Dilettant`, das im 18. Jahrhundert zur Benennung von Adligen erfunden wurde, die sich nicht-professionell - eben saus reiner Freude - mit Musik und bildender Kunst beschäftigten, haftet in der heutigen Verwendung oftmals eine pejorative Bewertung an: Dilettantismus wird mit Stümperei in Verbindung gebracht. «Laie» ist die neutralste der drei Bezeichnungen, doch können auch «Dilettant` und 〈Amateur in einer wertfreien Bedeutung auftreten. Letztlich scheint mir aber keine der genannten Bedeutungsnuancen ein Unterscheidungskriterium zu sein, mit dem man die drei Begriffe zuverlässig auseinanderhalten könnte (oder müsste), ohne sie unnötig und in künstlicher Weise von ihrem Alltagsgebrauch zu lösen. Sie alle - der Laie, die Amateurin, der Dilettant - können in ihrer Tätigkeit oder Kunst eine gewisse Fertigkeit erlangen, die sich jedoch vom meisterhaften oder professionellen Tun normalerweise immer noch - und zwar ganz spezifisch im Elaborationsgrad - unterscheidet. Als Gegenbegriffe gelten entsprechend «Experte`, «Fachmann/ Fachfrau〉, 〈Meister〉, 〈Könner〉 oder 〈Profi〉 (auch sie werden hier mehrheitlich austauschbar verwendet). Bedingungen für die Meisterschaft sind Ausbildung, Talent, technisches Know-how und weitere Elaborationskriterien, wie sie in Kapitel 1 genannt wurden, sowie meist auch eine berufliche - eben professionelle - Ausübung. Ab einem gewissen Niveau können Laien als «Autodidakten〉 oder 〈Semiprofis〉 gelten und wenn sie beginnen, ihre Tätigkeit gewerbsmäßig zu betreiben, kann die Grenze zum Profi überschritten werden. 
die Ideale der Elaboration und Perfektion aus Unkenntnis oder mangelhaftem Know-how unterwandert. Die «Rhetorik des Amateurs` spielt sich generell überall dort ab, wo Menschen im Gespräch zusammentreffen, d.h. in allen Formen der Alltagsrede, und im Speziellen auch dort, wo eine Rede von einer Person gehalten wird, die rhetorisch nicht - oder ungenügend - ausgebildet und geübt ist. Nicht jede Form der Laienrede ist fehlerhaft, doch ist sie meist deutlich weniger elaboriert als eine gekonnt vorbereitete und vorgebrachte Rede. Gerade in ihrer Ungeschliffenheit kann jedoch auch ein Reiz liegen.

Es ist ein zentrales Anliegen der klassischen Rhetorik, die Grenze zwischen gezielter Imperfektion, welche sich in einer bewusst belassenen sprachlichen Rauheit, in der Verbergung oder Herabsetzung der Kunst oder in figuraler Rede manifestiert, und dem echten Fehler, dem Vitium, in jedem Fall ziehen zu können: Die Rede eines ausgebildeten Orators, der die Kunst in allen Feinheiten beherrscht, soll jederzeit vom Redeschwall eines ungebildeten Redners und selbstverständlich auch von der gewöhnlichen Alltagsrede unterschieden werden können. Der Amateurredner ist das Gegenteil des Orator perfectus. Selbst die niedrigste der drei Stilhöhen (genus humile), die sich durch ihre Nähe zur Alltagssprache auszeichnet, wird immer noch durch klare Distinktionsmerkmale von der Laienrede abgehoben. Der Nutzen der rhetorischen Abweichung von der «einfachen und unbefangenen Sprechweise» wird auch darin gesehen, uns «vor der gewöhnlich-vulgären Sprechweise» zu bewahren (a volgari dicendi genere defendat) (Quint. inst. IX 3, 3). Trotz dieser deutlichen Zurückweisung der Laienrede anerkennen die antiken Rhetoriker durchaus auch Qualitäten, die sich aus der natürlichen und unvoreingenommenen Position des dilettantischen Redners oder alltäglichen Sprechers ergeben. Wie aber geht die klassische Rhetorik mit diesen positiven Seiten der Laienrede um, ohne das eigene Selbstverständnis in Frage stellen zu müssen?

\subsection{Die Alltagsrede: Der «natürliche» Redefluss}

\subsubsection{Die Umgangssprache als Vorbild}

Schon seit der Entstehung der Rhetorik findet sich auch der Einwand, dass die Ausbildung in Redekunst die natürliche Ausdrucksweise der Menschen verfremden könne. Vom «Gewöhnlichen» abzuweichen, um die Bewunderung des Publikums zu erwecken, ist laut Aristoteles ein zentrales Merkmal der poetischen Sprache. In der Rede dagegen könne der fremdartige Ton, der durch diese Abweichung vom normalen Ausdruck entstehe, einen Mangel bewirken (Aristot. rhet. III 2, 2, 1404b). Cicero sieht die Gefahr, dass rhetorische Bildung den Orator von 
seiner natürlichen Redegewohnheit abbringen könne, «die dem Geschmack des Volkes oder den Anforderungen des Forums entspricht» (Cic. de orat. I 81). Auch wenn die Ausdrucksweise durch das Rhetorik-Studium schön und das Gesagte eindrucksvoll werde, wirke der gebildete Stil «so glänzend und üppig», dass er mehr an Wettkampfplätze und Salböl erinnere als an die gewöhnliche Welt der öffentlichen Versammlungen und des Forums (Cic. de orat. I 81). Auch Quintilian gesteht ein, «daß die Bildung auch etwas nimmt, wie die Feile dem Rauhen, der Schleifstein dem Stumpfen und dem Wein das Alter» (Quint. inst. II 12, 8). Das Raue, Ungeschliffene hat in der Rede eine Qualität, die ihr durch Bildung bzw. Ausfeilung genommen wird. Letztlich hält Quintilian den positiven Aspekt der Ungeschliffenheit jedoch für geringer als den Fehler, der durch das Schleifen (perpolire) entfernt werde (Quint. inst. II 12, 8). Neben den genannten Einwänden scheint zu Quintilians Zeit auch substanziellere Kritik an der rhetorischen Ausbildung laut geworden zu sein:

Weiter meinen manche, es gäbe keine natürliche Beredsamkeit außer der Sprache, die der des Alltags am ähnlichsten sei, in der wir mit unseren Freunden, Gatten, Kindern und Sklaven sprechen, die sich begnügt, unsere Willensmeinung auszudrücken, und nichts Gesuchtes [arcessitus] und mühsam Ausgearbeitetes [elaboratus] verlangt (Quint. inst. XII 10, 40).

Diese rhetorikskeptische Sichtweise wird durch die Behauptung begründet, «der ungepflegte Redestrom, wie ihn das Geratewohl mit sich bringt, entspreche mehr der Natur oder wohl gar echter Mannesart» (Quint. inst. IX 4, 3). Argumentiert wird hier mittels der Natürlichkeit der Alltagsrede und der Spontanität, mit der sie sich ergibt. Quintilian selber verwehrt sich jedoch gegen eine solche Auffassung. Denn sie würde ja all das, was seine Rhetoriktheorie über die Möglichkeiten der Elaboration und das Ziel der Perfektionierung der Rede sagt, fundamental in Frage stellen: das akribische Bemühen des Redners, seine Suche nach glanzvollen Stilmitteln, die überlegte Vorbereitung, die kunstvolle Ausarbeitung und die figurale Abweichung von der alltäglichen Redepraxis. Quintilian kontert die Kritik geschickt, indem er der Rhetorik selbst eine «naturgemäße» Art der Kultivierung der Rede attestiert. Er stützt sich dabei auf das Argument der Nützlichkeit und Wirksamkeit einer gekonnt kultivierten Rede und so in gewissem Sinn auch auf die Idee einer 〈zweiten Natur〉, wie wir sie beim Kulturland oder Nutzvieh finden: «In Wahrheit ist aber doch das am meisten naturgemäß [maxime naturale], was die Natur sich am besten entfalten läßt» (Quint. inst. IX 4, 5). Die Naturanlage kann, wie sich mit Cicero ergänzen lässt, durch die - stets an der Natur orientierte - Kunst und fleißiges Üben gesteigert und zur Vervollkommnung gebracht werden (Cic. de orat. II 150; vgl. auch Neumann 2003: 144-146). 
Da die Redekunst laut Cicero in der naturgegebenen Anlage des Sprechens verankert ist, bestimmt sich Expertise in der Rhetorik ein wenig anders als in den anderen Künsten. Während man bei den übrigen Künsten «meist aus entlegenen, versteckten Quellen» schöpfe, läge die Kunst der Rede «vor aller Augen», werde «ganz allgemein geübt» und von allen Menschen «im Mund geführt» (Cic. de orat. I 12). Nicht nur liege der Fundus der Redekunst in der täglichen Redepraxis, sondern die Art und Weise, wie wir im Alltag sprächen, solle auch als Maßstab für die kunstvoll gestaltete Rede gelten. Während in anderen Künsten das besonders hervorrage, «was von der Einsicht und Denkweise der Laien am weitesten entfernt ist», wäre es «beim Reden ein massiver Fehler [...], gegen die übliche Ausdrucksweise und die Gewohnheit des allgemeinen Empfindens zu verstoßen» (a vulgari genere orationis atque a consuetudine communis sensus abhorrere) (Cic. de orat. I 12). Laut Auctor ad Herennium lassen sich zudem die Tugenden der Deutlichkeit und der Elegantia in einer Rede am besten erfüllen, indem eine gebräuchliche Wortwahl verwendet werde, die der täglichen Umgangssprache entnommen sei (Rhet. Her. IV 12, 17). Demetrios fordert sogar, die für eine Rede gewählte Sprache solle sich die gewöhnliche Art zu sprechen zum Vorbild nehmen:

Wie für alles andere, so besonders für Metaphern ist die Umgangssprache Lehrerin. Denn fast alles überträgt sie unbemerkt, weil sie es sicher und ohne Anstoß tut: sie nennt eine Stimme helleuchtend, einen Menschen scharf, eine Sitte rauh, einen Redner groß, kurz: alles derartige überträgt sie so geschmackvoll, daß dies den Eindruck macht, den ursprünglichen Ausdrücken ähnlich zu sein. (Demetr. eloc. II 86)

Die «natürliche Gewandtheit der Umgangssprache» soll deshalb als Richtschnur gelten für die Verwendung von Metaphern in Prosa und Rede (Demetr. eloc. II 86). Nicht nur ihre natürliche Sicherheit hat die Alltagsrede der Rhetorik laut Demetrios voraus, sondern auch jene Subtilität der Übertragungen, welche fast unbemerkt die Sprache durchziehen, sodass sie bald nicht mehr als uneigentliche Rede wahrgenommen würden: «So schön hat jedenfalls einiges die Sprache des Volkes übertragen, daß wir die ursprünglichen Ausdrücke nicht mehr brauchen. Die Metapher ist geblieben und behauptet den Platz des eigentlichen Ausdrucks, z.B. das Auge der Rebe» (Demetr. eloc. II 87). Aristoteles sieht die Metapher gar als das einzige Stilmittel, das neben dem «Wort in seiner vorherrschenden und eigentümlichen Bedeutung» für die Rede geeignet sei, und zwar deshalb, weil nur Metaphern von allen Menschen gebraucht würden (Aristot. rhet. III 2, 6, 1404b). Auch hier gilt die natürliche Art des Sprechens als Maßstab dafür, was in einer Rede zulässig ist und was nicht.

Selbst Quintilian, der stärker dem Ideal der kunstvollen Ausarbeitung der Rede verhaftet ist, sieht den Ursprung der Übertragung oder Translatio, die er für den häufigsten sowie die den mit Abstand schönsten Tropus hält, in unserem 
alltäglichen Umgang mit der Sprache. Die Metapher sei uns «schon von der Natur selbst so weit zueigen gemacht, daß auch Menschen ohne Schulung und ohne es zu merken oft von ihr Gebrauch machen» (Quint. inst. VIII 6, 4). Cicero stellt ebenfalls fest, dass die Translatio als Möglichkeit, «ein Wort in übertragener Bedeutung zu gebrauchen», weit im Volk verbreitet sei (Cic. de orat. III 155): «Denn daß «die Reben Augen treiben, die Saaten üppig grünen und die Felder prangen», das sagen auch die Bauern» (Cic. de orat. III 155). Neben der Translatio erwähnt Quintilian eine Reihe weiterer Tropen, die in der Alltagssprache verwurzelt seien. So könne die Parabel bzw. der Vergleich «jedermann auch in der Alltagssprache begegnen» (Quint. inst. VIII 3, 81). Desgleichen bringe die Synekdoche nicht nur als Schmuck Ablenkung in die Rede, sondern sei auch eine gebräuchliche Form der Alltagssprache (cotidiani sermonis usus), «so daß wir bei einem Ding an mehrere denken, bei einem Teil an das Ganze, bei der Art an die Gattung, bei dem Vorausgehenden das Folgende oder auch bei allem umgekehrt» (Quint. inst. VIII 6, 19-21). Auch die Umkehrung (inversio), d.h. die Darstellung eines Wortlauts, «der entweder einen anderen oder gar zuweilen den entgegengesetzten Sinn hat», sei eine Figur, die «geringen Talenten und der Alltagssprache oft genug zu Diensten» stehe (Quint. inst. VIII 6, 44; 51). In alltäglichen Situationen geläufige Allegorien wie «auf den Leib rücken» oder «an die Kehle gehen» würden uns laut Quintilian nicht einmal mehr besonders auffallen. Aufgrund einer übertriebenen Jagd nach neuartigen, effektvollen, überraschenden und Genuss verschaffenden Ausdrücken durch die Redner hätten viele solche Ausdrücke allerdings ihren besonderen Reiz für die Rede verloren (Quint. inst. VIII 6, 51).

Die Gefahr, sich in der Rede zu stark von der Umgangssprache abzusetzen, erfolgt laut Quintilian aus dem eigentlich lobenswerten Impuls, die unanständige, schmutzige oder auch einfach nur niedrige Rede zu meiden: «Niedrig aber klingt, was unter dem Rang ist, der dem betreffenden Gegenstand oder der Person zukommt. In dem Bestreben, vor diesem Fehler auf der Hut zu sein, pflegen manche erst recht in die Irre zu gehen, die vor allem zurückschrecken, was zur Umgangssprache gehört, auch wenn es im Interesse des Falles unentbehrlich ist» (Quint. inst. VIII 2, 2). Was zu weit vom Alltagsgebrauch abweicht, kann den Redner prahlerisch erscheinen und die Rede umständlich werden lassen: Anstatt eitel von «iberischen Gräsern» zu sprechen, was niemand verstehe, solle man ein Binsenseil doch einfach beim Namen nennen, statt «in Salzbrühe dauerhaft gemachte Fische» zu sagen, bezeichne man diese besser kurz und trivial als «Salzfische» (Quint. inst. VIII 2, 2-3). 


\subsubsection{Der Reiz des Ungekünstelten}

Schon Aristoteles rät dem Redner - ganz im Sinne der oben beschriebenen Dissimulatio Artis -, beim Sprechen unauffällig ans Werk $\mathrm{zu}$ gehen und keinen gekünstelten, sondern einen natürlichen Eindruck zu erwecken, damit bei den Zuhörern nicht der Eindruck entstehe, er führe etwas gegen sie im Schilde. Um diese «natürliche〉 Wirkung zu erreichen, empfiehlt Aristoteles die Verwendung umgangssprachlicher Wörter (Aristot. rhet. III 2, 4-6, 1404b). Die allen Personen 〈von Natur aus〉 gegebene Umgangssprache wird so zum Mittel der Vertuschung der Kunst. Worin aber zeigt sich die Natürlichkeit des umgangssprachlichen Stils, das Gewöhnliche der Alltagsrede? Quintilian hält eine abgerissene (abruptus) und zerhackte (dissolutus) Sprechweise für ein Kennzeichen der Laienrede (Quint. inst. II 11, 6-7) Ebenso charakteristisch für den mündlichen Stil der Alltagssprache sei das Anakoluth, d.h. ein unmotivierter Wechsel oder eine Inkonsequenz in der Satzkonstruktion, und auch eine gänzlich arhythmische Art zu sprechen wirke «ungebildet und bäurisch» (Quint. inst. IX 4, 56). Um die damit verbundene Natürlichkeit auszustrahlen, finden sich schon in der Antike Redner, die sich an die holprige Redeweise der Laien angleichen: Die Kunst eines Lysias besteht laut Quintilian gerade darin, die Worte so zu verbinden, als wären sie «urwüchsig und ungefüge» (rudis, incompositus) (Quint. inst. IX 4, 17). Mit einer üppigen Rhythmisierung dagegen hätte Lysias den «Reiz der einfachen, ungekünstelten Färbung» (gratia simplicis atque inadfectati coloris) und damit auch seine Glaubwürdigkeit eingebüßt (Quint. inst. IX 4, 17). Die Taktik, sich in der Redekunst das natürliche, unaffektierte Gefüge und den Klang der simplen, unverfälschten Sprache zu eigen zu machen, ist somit altbewährt. Die Natürlichkeit des Redeflusses zeigt sich Cicero zufolge auch in den Atempausen, die durch die Notwendigkeit des Luftholens wie von selbst entstünden. Er stellt eine enge Beziehung her zwischen der natürlichen Zweckmäßigkeit des Atemrhythmus und dem Wohlklang, der daraus für unsere Ohren entstehe:

Denn Pausen und Einschnitte bringt ja das Luftholen und die Kürze unseres Atems mit sich. Diese Erfindung ist jedoch so angenehm, daß wir auch dann, wenn jemand einen unbegrenzten Atem hätte, doch nicht wollten, daß er ununterbrochen redete. Denn wir erfahren als angenehm für unser Ohr, was für die Lungenkraft des Menschen nicht nur erträglich, sondern leicht zu leisten ist. (Cic. de orat. III 181)

Der natürliche Atemrhythmus, der auch dem Alltagsredner vorgegeben ist, scheint nun jedoch nicht ausreichend $\mathrm{zu}$ sein, um den Redefluss angemessen zu temperieren: «Der Laie sprudelt regellos [incondite] hervor, soviel er kann, und findet das Maß dessen was er sagt, in seinem Atem, nicht in seiner Kunst; der Redner aber kleidet den Gedanken so in Worte, daß er ihn gewissermaßen 
in einem sowohl freien wie gebundenen Rhythmus erfaßt» (Cic. de orat. III 175). Im Vergleich zur Dichtung solle die Rede freier und gelöster sein, «doch nicht in der Art, daß sie sich verflüchtigt und verirrt, sondern so, daß sie sich auch ohne Fesseln selbst das rechte Maß zu setzen weiß» (sibi ipsa moderetur) (Cic. de orat. III 184). Durch ihre lockere, durch Pausen gegliederte Rhythmisierung und die ausgewogene Proportionierung soll sich die kunstvolle Rede laut Cicero daher vom natürlichen Redefluss der Laien abheben: «Denn wenn man jene pausenlose, ununterbrochen plätschernde Geschwätzigkeit für plump und ungeschliffen [rudis et impolita] halten muß, was gibt es dann für einen anderen Grund, sie zurückzuweisen, als den, daß das Gehör der Menschen selbst zum Klang der Stimme unwillkürlich den Takt schlägt?» (Cic. de orat. III 185).

\subsubsection{Die Kraft des Ungehobelten}

Und doch kann, wie Quintilian beobachtet, die grobschlächtige Art zu sprechen, wie sie vielen rhetorisch Ungebildeten zu eigen ist, zumindest auf ein unerfahrenes Publikum mächtiger wirken als die geschliffene Redekunst, «denn die klare Gliederung, so entscheidend sie auch für die Prozeßrede ist, mindert doch den Anschein der aufgewendeten Kraft, das Ungehobelte wirkt größer als das Geglättete [rudia politis maiora], das Ausgestreute reicher an Zahl als das Wohlgeordnete» (Quint. inst. II 12, 3). Die grobe Redeart, zu der auch rückhaltloses Schimpfen und überhaupt eine gewisse Ungeniertheit gehöre, erwecke den Anschein grosser Redekraft, und selbst das Übersteigerte könne auf die Zuhörer großartig wirken (Quint. inst. II 12, 4-5): "Auch deshalb scheinen bisweilen die Ungebildeten den größeren Reichtum [copia] zu besitzen, weil sie alles in den Mund nehmen, während die Gebildeten auswählen und maßhalten» (Quint. inst. II 12, 6).

Was die Beurteilung der Kraft der Laienrede angeht, zeigt sich erneut Quintilians ambivalente Haltung. Einerseits attestiert er Bauern und nicht ausgebildeten Rednern Sprachgewalt aufgrund ihres ungeschliffenen Stils, andererseits stuft er die Sprechweise dieser Laienredner als minderwertig herab. Um das Dilemma aufzulösen, bedient sich Quintilian eines rhetorischen Kniffs: Die vermeintliche Kraft (vis), die durch die Ungeschliffenheit der Rede entstehe, wird von ihm als Gewalttätigkeit (violentia) entlarvt und somit als unwürdig erwiesen (Quint. inst. II 12, 11). An anderer Stelle stellt Quintilian die Wirksamkeit der Amateurrede in Frage: «Wie aber kann Ungeordnetes eine stärkere Wirkung haben, als was fest gebunden und richtig an seinen Platz gestellt ist?» (Quint. inst. IX 4, 6). Erst ein zusammenhängender Redefluss, der ohne Hindernisse fließen könne, entfalte seine ganze Kraft - was beim gebrochenen oder abgehackten Strom der ungenügend ausgestalteten Rede nicht der Fall sei (Quint. inst. IX 4, 6-7). In Quintili- 
ans Augen schwächt die schöne und geschliffene Erscheinung die Kraft der Rede nicht, sondern diese könne erst durch die kunstvolle Ausarbeitung richtig zur Geltung kommen (Quint. inst. IX 4, 7). Am Ende stellt Quintilian die Kunst über das unelaborierte Sprechen.

\subsubsection{Subabsurdus: Der Alltagswitz}

Humor ist eines der wenigen Wirkungsmittel der Rede, das in allen möglichen Situationen auftreten kann und das auch unsere Alltagsrede durchzieht - was schon Cicero feststellt: «In der Tat sind ja auch alle Proben des Witzes, die ich zu behandeln habe, nicht so sehr eine Würze öffentlicher Verhandlungen wie aller Äußerungen überhaupt» (Cic. de orat. II 271). Ja, es gebe eigentlich keinen Lebensumstand, in dem Charme und Gewitztheit unangebracht wären (Cic. de orat. II 272). Dazu gehörten auch «die etwas ungereimten [subabsurdus], aber gerade dadurch erheiternden Äußerungen», die kein Ausdruck von subtilem Witz (urbanitas) seien, sondern einem Alltagshumor entsprängen, der die Dinge nicht immer passend beschreibe oder sich auch einmal an der Grenze des guten Geschmacks bewegen könne (Cic. de orat. II 274). Eine gewisse Derbheit und Simplizität charakterisiert somit den Alltagswitz. Kippt der Humor z.B. aufgrund einer Zweideutigkeit ins allzu Vulgäre oder gar Obszöne, sei dies in einer Rede jedoch klar zu tadeln (Quint. inst. VI 3, 47).

Insgesamt beobachtet Quintilian auch beim Witz, dass es vielen ungeschulten Rednern aufgrund ihrer natürlichen Veranlagung und eines Gespürs für den passenden Stoff gelinge, die witzige Redeweise gekonnt vorzubringen. Außerdem bemerkt er: «manche Menschen besitzen einen eigentümlichen Reiz in ihrer Haltung und Miene, so daß die gleichen Bemerkungen, wenn sie ein anderer machte, weniger witzig und fein wirkten» (Quint. inst. VI 3, 12). Weil die passende Gelegenheit für den Witz eine wesentliche Rolle spiele, komme es, «daß mit Hilfe günstiger Umstände nicht nur ungeschulte, sondern auch ganz und gar ungehobelte Menschen witzig sprechen» (Quint. inst. VI 3, 13). Aus diesen Gründen treffe man bei gemeinsamen Mahlzeiten oder alltäglichen Unterhaltungen immer wieder schlagfertige Menschen an, die durch den täglichen Umgang miteinander im Witzemachen sehr geübt seien (Quint. inst. VI 3, 14). Desgleichen sei die Übertreibung (hyperbole), welche Gelächter errege, «unter Ungebildeten und bei Bauern» allgemein verbreitet, was «verständlich genug» sei, «denn von Natur liegt in allen Menschen das Verlangen, die Dinge zu vergrößern oder zu verkleinern, und niemand gibt sich mit dem zufrieden, wie es wirklich ist» (Quint. inst. VIII 6, 75). 
Cicero geht davon aus, dass es keine Regeln des Witzes gebe, weshalb Humor nicht eigentlich lehrbar sei, sondern vielmehr «besondere Gaben der Natur» voraussetze (Cic. de orat. II 216). Zwar seien beim Witz die beteiligten Personen, das Thema sowie der Zeitpunkt als Faktoren der jeweiligen Redesituation zu berücksichtigen - was ja eine generelle Forderung des Aptum ist -, doch bedeute dies vor allem, witzige Bemerkungen dort zu unterlassen, wo sie keinen Dienst erweisen. Ansonsten ließen sich jedoch kaum Anweisungen zum Witz formulieren (Cic. de orat. II 229). Humor und Schlagfertigkeit sind also Redemittel, die nicht den ausgebildeten Rednern mit ihrer ausgewiesenen rhetorischen Expertise vorbehalten sind, sondern die gerade Personen aus dem einfachen Volk besonders wirkungsvoll einzusetzen wissen. Gleichzeitig ist jede Form von Vulgarität, die den Alltagswitz oft kennzeichnet, aus Sicht der der klassischen Rhetorik unbedingt zu verurteilen.

\subsection{Vulgaris orator: Der Laienredner als ungeschliffene Persönlichkeit}

Wie aber schätzt die klassische Rhetorik die rednerischen Fähigkeiten und die Ausstrahlung des nicht rhetorisch gebildeten Redners selbst ein? Die Antwort hängt unter anderem davon ab, welcher Status dem rhetorischen Lehrsystem zugestanden wird. Cicero, welcher der rhetorischen Unterweisung kritisch gegenübersteht und vom Redner primär eine gute Allgemeinbildung und ein in der Philosophie geschärftes Denken fordert, attestiert dem Redner ohne Ausbildung eine natürliche Kunstfertigkeit und hebt verschiedene Qualitäten seiner spontanen Redefähigkeit hervor. Quintilian ist gegenüber dem unkultivierten Redner grundsätzlich kritischer eingestellt, da er - wie schon der Titel seines Hauptwerks zeigt - die Institutio bzw. 〈Ausbildung〉 des Redners für unabdingbar hält. Doch zeigt er sich auch in diesem Punkt erstaunlich ambivalent. Neben dem offensichtlichen Unvermögen des unausgebildeten Redners sollen im Folgenden daher auch seine positiven Eigenschaften betrachtet werden.

\subsubsection{Natürliche Eloquenz bar jeder Zurschaustellung}

Im Gegensatz zu anderen Künsten wie der Musik oder der Geometrie hält Cicero es im Bereich der Redekunst für praktikabel, dass selbst ein ungebildeter, gewöhnlicher Redner (vulgaris orator) es zu einer gewissen Anerkennung bringen kann (Cic. de orat. III 79). Bei jeder Rede liege nämlich sowohl die Würde als auch die Fülle des Ausdrucks bereits im Stoff, von dem die Rede sei, so dass ihr Glanz sich aus dem jeweiligen Inhalt ergebe (Cic. de orat. III 125). Mit ein wenig Allgemeinbildung, 
Übung und Begabung könne ein jeder die richtigen Worte finden, ohne Instruktionen von Redelehrern darüber zu benötigen, wie die Worte anzuordnen oder ins rechte Licht zu rücken seien: «So einfach findet die Natur selbst ohne Führer, wenn sie nur geübt ist, im Überfluß des Stoffs den Weg zum Schmuck der Rede» (Cic. de orat. III 125). Cicero hält gar lobende Worte bereit für die natürliche Art, in der gewöhnliche Menschen im Alltag sprechen, wie etwa für Crassus' Schwiegermutter Laelia: «Sie ist schon im Klang ihrer Stimme so schlicht und einfach, daß nichts den Eindruck von Schaustellung oder Nachahmung erweckt» (nihil ostentationis aut imitationis adferre videatur) (Cic. de orat. III 45). Das Unprätentiöse einer nicht auf Wirkung ausgerichteten Redepraxis wird hier lobend hervorgehoben.

Um der bescheidenen und zugleich dem Publikum schmeichelnden Wirkung willen hält Quintilian denn auch den gebildeten Redner dazu an, die Sprache seiner Rede nahe beim Volk zu halten und sich als Einheimischer zu erkennen zu geben (Quint. inst. VIII 1, 2-3). Keinesfalls dürfe die Anpassung der Sprache an die jeweilige Region dabei jedoch ostentativ zelebriert werden. Was sonst geschehen kann, zeigt Quintilian in einer Anekdote, in welcher dem großen Redner und Wahl-Athener Theophrast nach seiner Rede von einer alten Attikerin der Spiegel vorgehalten wird. Diese wirft Theophrast nämlich vor, er habe mit allzu großer Sorgfalt ein attisches Wort immer wieder in seine Rede eingestreut, so dass er sich gerade dadurch als «Gast von auswärts» zu erkennen gegeben habe (Quint. inst. VIII 1, 2-3). Wie Quintilian aus diesem Lehrstück schließt, solle ein Redner sich nur «durch einen Anhauch» als Bewohner einer Stadt zu erkennen geben und nicht so, als sei ihm «das Bürgerrecht verliehen worden» (Quint. inst. VIII 1, 2-3). Der grundsätzlich wünschenswerte Annäherungseffekt wird durch übertriebene Beflissenheit, eine überangepasste, die einheimische Umgangssprache noch übertreffende Sprache wieder zunichte gemacht.

\subsubsection{Warum gelten gemeinhin die Unstudierten als begabter?}

Viele ungebildete Redner würden sich damit brüsten, ihrem «eigenen Schwung» $\mathrm{zu}$ folgen und sich «ihrer eigenen Kraft» zu überlassen, wie Quintilian bemerkt (Quint. inst. II 11, 3-12). Daher sei auch die Vorstellung verbreitet, die Nichtstudierten könnten mit besonderen Gedankenblitzen und Pointen auftrumpfen, wirkten beim Reden weniger gehemmt und seien überhaupt begabter als die studierten Redner (Quint. inst. II 11, 3-12). Quintilian hält dies jedoch für ein Fehlurteil, «als sei da die Kraft größer, wo die Kunst fehle, wie wenn man etwa glaubt, Aufbrechen sei gegenüber dem Öffnen, Zerreißen gegenüber dem Loslösen, Wegziehen gegenüber dem Führen das Zeichen größerer Stärke» (Quint. inst. II 12, 1). Wir sehen hier erneut, wie Quintilian zwischen echter rhetorischer Kraft 
und roher Gewalt unterscheidet - und dem ungebildeten Redner allenfalls die Kraft des Gewalttätigen zubilligt. Insbesondere stößt Quintilian sich daran, dass Redner, die in der Rhetorik nicht ausgebildet seien, sich beim Reden nicht um ein organisches Ganzes bemühten, denn sie «schleudern in abgerissenen Stücken heraus, was ihnen gerade unter die Hände kommt. So kommt es, daß eine zerhackte und aus Verschiedenartigem zusammengestückte Rede keinen Zusammenhang haben kann» (Quint. inst. II 11, 6-7). Auch die Redemittel würden allein zur (meist schlechten) Unterhaltung der Ohren der Anwesenden angewendet und die verwendeten Sentenzen leuchteten vor allem deshalb besonders hervor, weil um sie herum «Schmutz und Unrat» sei (Quint. inst. II 12, 6-7). Überhaupt hält Quintilian die Verwendung von Sentenzen, d.h. einprägsamen, belehrenden Sätzen, nicht für ein Stilmittel, das «allenthalben und von jedem Beliebigen» gesprochen werden sollte (Quint. inst. VIII 5, 7). Sentenzen schickten sich ausschließlich für Personen, die Ansehen genießen (in quibus est auctoritas), da sie durch ihre Person das Gewicht der Sache bestätigten: «Denn wer könnte einen Knaben, einen jungen Mann oder auch einen gewöhnlichen Menschen ertragen, wenn er in seinen Worten mit uns ins Gericht ginge und gewissermaßen den Lehrer spielte?» (Quint. inst. VIII 5, 8).

Quintilian zeichnet ein negatives Bild des Laienredners, welcher mit seiner gewaltsamen, effekthascherischen oder belehrenden Art und einem abgehackten, zusammenhangslosen Redestil die Redekunst verunglimpfe. Selbst Cicero, der dem Orator vulgaris eine gewisse Eloquenz und Unverfälschtheit zubilligt, räumt ein, dass der ungebildete Redner, verglichen mit einem wahrhaft perfekten Redner, der philosophisch umfassend ausgebildet und in der rhetorischen Praxis bewandert ist, stets kraftlos und ungeschliffen wirken werde:

Denn einerseits kann man als Redner nicht genügend Energie und Durchschlagskraft besitzen, ohne durch öffentliche Tätigkeit gestählt zu werden, andererseits hat man aber auch nicht genügend Schliff und Wissen [satis politus et sapiens], ohne mancherlei studiert zu haben. (Cic. de orat. III 80)

\subsubsection{Menschlichkeit und Emotionalität des Spontanen}

Vielleicht ist Quintilian auch deshalb etwas gehässig gegenüber den ungebildeten Rednern, weil sie im Gegensatz zu seinen Zöglingen der Gefahr der Verkrampfung entgehen, welche eine rhetorische Ausbildung mit ihren hohen Anforderungen an Sorgfalt, Überlegung, Haltung und Fleiß mit sich bringen kann. Scheinen Leichtigkeit und Spontaneität im Reden bei Laien noch unverstellt vorhanden zu sein, läuft der gebildete Redner Gefahr, gerade diese positiven Eigenschaften 
zu verlieren. Der Hang zur Erstarrung zeigt sich bereits bei der Konzeption einer Rede. Um locker zu bleiben, empfiehlt Quintilian seinen Zöglingen, beim Verfassen ihrer Rede nicht allzu angestrengt zu suchen oder sich gar stundenlang abzuquälen, sondern das Schreiben lustvoll und mit einer gewissen Leichtigkeit anzugehen. Sie sollten kurz überlegen, was die zu behandelnde Sache erfordere, was für die Person und die Situation passe und sich so «ans Schreiben machen, wie es sich für einen vernünftigen Menschen gehört» (Quint. inst. X 3, 15). Die meisten Dinge würden sich wie von selbst aus der Natur der Sache ergeben und ins Auge fallen, «deshalb suchen ja auch die Ungebildeten und die einfachen Bauern nicht lange, womit sie anfangen sollen. Um so beschämender ist es daher, wenn die Schulbildung es schwerer macht» (Quint. inst. X 3, 16).

Noch deutlicher als bei der Vorbereitung der Rede wird der Faktor Spontaneität in der Actio, also beim Vortrag, sichtbar. Wie bereits angesprochen, kann eine scheinbar mühelos hingeworfene Rede Sympathie und Bewunderung beim Publikum ernten. Cicero hält es nicht nur für ein Zeichen von Esprit, sondern auch von Menschlichkeit, wenn der Redner bisweilen eine spontane Reaktion zeige. So würden beim Publikum Äußerungen, mit denen ein Redner auf eine Herausforderung reagiere, besser ankommen als solche, mit denen er anderen zuvorkomme: «Denn einerseits ist die Schlagfertigkeit [ingeni celeritas], die bei der Antwort in Erscheinung tritt, noch größer, und andererseits ist es menschlich, daß man reagiert» (humanitatis est responsio) (Cic. de orat. II 230).

Unausgebildete wie ausgebildete Redner können in die Lage kommen, auf eine unvorhergesehene Situation reagieren oder gar spontan und unvorbereitet bzw. 〈aus dem Stegreif eine Rede halten zu müssen. Es stellt sich somit die Frage, «ob nicht speziell die facultas subito dicendi ohne Artifizialität möglich ist oder sogar wirksamer» sei als jede vorbereitete Rede (Geißner 2007: Sp. 1362). Ganz ohne Künstlichkeit ist, wie wir bereits gesehen haben, in der klassischen Rhetorik auch die Stegreifrede nicht denkbar. Laut Quintilian unterscheidet sich eine gekonnte Stegreifrede klar vom «zufälligen Redeerguss» Ungebildeter, «den wir ja auch, wenn Weiber sich zanken, im Überfluß erleben können» (Quint. inst. X 7, 13). Aus seiner Abneigung gegen jegliches unreflektierte «Wortgetümmel» heraus verlangt Quintilian von gebildeten Rednern, dass sie selbst in überraschenden Situationen imstande seien, durch Routine und eingeübte ad-hoc-Strategien eine gewisse Überlegung, Ordnung und Ausschmückung in ihre Rede hineinzubringen (Quint. inst. X 7, 8-13; vgl. Kap. 2.3.3). Dennoch ist selbst Quintilian in dieser Angelegenheit hin- und hergerissen. Er gesteht nämlich ein, dass Laienredner oder gänzlich unvorbereitete Redner eine Wärme und Begeisterung in ihrer Stegreifrede $z u$ entfalten vermöchten und damit einen Erfolg beim Publikum erzielen könnten, der durch sorgfältige Vorbereitung nicht zu erreichen wäre (Quint. inst. $\mathrm{X} 7,13)$ : 
Denn die gut erfaßten Gefühlswirkungen und die ganz frischen Vorstellungen von den Gegenständen, über die wir reden, reißen hier in einem einheitlichen Schwung mit, während sie zuweilen durch die Verzögerung bei der Niederschrift sich abkühlen und, wenn sie hinausgeschoben werden, nicht wiederkehren. (Quint. inst. X 7, 14)

Gerade für das Transportieren von Emotionen scheint Unmittelbarkeit also von zentraler Bedeutung zu sein. Durch eine «unergiebige Wortklauberei» bei der Vorbereitung dagegen könne die mitreißende Redegewalt verloren gehen und die Rede zu bloßem Stückwerk verkommen (Quint. inst. X 7, 14). Die Fähigkeit zum anschaulichen und gefühlvollen Sprechen hält Quintilian für allen Menschen gleichermaßen gegeben, da diese der menschlichen Vorstellungskraft entspringe: «Und deshalb fehlen auch denen, die gar keine Ausbildung genossen haben, die Worte nicht, wenn sie nur von einer Leidenschaft ergriffen sind» (Quint. inst. X 7, 15). Starke Affekte, aber auch der Druck des Moments oder die Angst vor einer Blamage könnten einen Stegreifredner unter Umständen regelrecht beflügeln - so dass er sich in die Lage versetzt sehe, bestimmte Gedanken in einer Weise hervorzubringen, wie es ihm normalerweise nur schwer glücken würde (Quint. inst. X 7, 16-17). Aus all diesen Gründen, so Quintilian, müsse eine im Voraus überdachte Rede nicht zwingend besser sein als eine Stegreifrede. Und doch sei die vorbereitete Rede mit deutlich weniger Gefahr verbunden. Der Erfolg, der einer spontanen Rede hin und wieder beschieden sei, dürfe den gebildeten Redner keinesfalls zu Nachlässigkeit verleiten (Quint. inst. X 7, 19). Selbst eine Stegreifrede sollte für Quintilian - gemessen an dem, was ohne Vorbereitung geleistet werden könne - vollkommen (perfectus) sein (Quint. inst. X 7, 28). Damit überwiegen bei Quintilian auch in Sachen Stegreifrede am Ende die Vorzüge der Sorgfalt und Perfektion über die Qualitäten des Unmittelbaren und Spontanen.

\subsection{Purus sermo: Die Stilhöhe des Gewöhnlichen}

Die Rhetoriktheorie der Antike behandelt vornehmlich Reden in öffentlicher Funktion, wie etwa die Gerichtsrede oder die Beratungsrede vor dem Senat. Nur wenig findet sich in der klassischen Rhetorik dagegen über den Stil alltäglicher Redesituationen. Allgemein wird jedoch im Sinne des Aptum geraten, die Stilhöhe einer Rede der Wichtigkeit und Bedeutsamkeit des behandelten Gegenstands und an die Umstände anzupassen. Bei weniger wichtigen Themen sowie in unbedeutenden oder alltäglichen Situationen solle die Stilhöhe entsprechend niedrig gehalten werden. 


\subsubsection{Man soll sich nicht um die Regenrinne ereifern: Niedriger Stil für einfache Fälle und Alltagsgespräche}

Quintilian zufolge sind schmuckvolle, erhabene Worte zwar wirkungsvoll, doch passen sie nicht unbedingt zu jedem Gegenstand: «Ein Wort, das hier prächtig wirkt, wirkt an anderer Stelle geschwollen, und Wörter, die bei großen Gegenständen niedrig wirken, wirken bei kleineren passend» (Quint. inst. VIII 3, 18). Das Aptum kann eine Senkung der Stilhöhe verlangen, damit sich die Rede an die unbedeutende Thematik anpasst. So verlangten zwar offizielle Senatsreden nach einem erhabenen Ton und Gerichtsreden nach einer sorgfältigen Redeweise; auch eine Volksrede werde passenderweise in einem erregteren Ton vorgebracht (Quint. inst. VIII 3, 14):

Dagegen dürfte sich für eine private Beratung [privatum consilium] und für Fälle, bei denen, wie es so häufig geschieht, nur wenig Geld auf dem Spiele steht, ein reiner Gesprächston [purus sermo], der die Sorgfalt verbirgt [dissimilis curae], eher sich schicken. Oder sollte man sich nicht schämen, eine bestimmte Schuldsumme in schwungvollen Perioden zu fordern oder sich um die Regenrinne zu ereifern? (Quint. inst. VIII 3, 14)

Ein ähnliches Beispiel bringt Cicero vor, um das Indecorum zu illustrieren: «Wie unpassend ist es doch, etwa im Zusammenhang mit Dachrinnen vor einem einzigen Richter großartige Wörter und allgemein gehaltene Überlegungen vorzubringen» (Cic. orat. 21, 72). In Fällen, in denen es nur um Kleinigkeiten gehe, sei auf die «Feuerbrände der Beredsamkeit» zu verzichten, «damit wir nicht Spott oder Abscheu zu verdienen scheinen, wenn wir bei Nichtigkeiten Tragödien aufführen» (Cic. de orat. II 205). Kleinere Prozessfälle sowie auch persönliche Gespräche oder Briefe fordern laut Quintilian zudem eine weniger gebundene oder verwobene Form und einfachere Rhythmen als die offizielle Rede (Quint. inst. IX 4, 19-21). Cicero erlaubt für den privaten Briefwechsel, den er mit einem persönlichen Gespräch mit einem Freund vergleicht, sogar den «plebeius sermo», also den gewöhnlichen, im Volk verbreiten Plauderton (Cic. Att. XII 39, 2; vgl. Pompe 2003: Sp. 186). Im Alltag und in trivialen Fällen bietet sich ein gewöhnlicher und emotionslos gehaltener Redestil an - und wo die Rede vorbereitet wurde, sollte die aufgewendete Mühe besonders gut verborgen werden.

\subsubsection{Ornatus purus: Schlichtheit als Schmuck und Merkmal moralischer Integrität}

Handelt es sich um unwichtige Fälle, erscheint Quintilian denn auch die «Einfachheit [simplicitas] und gleichsam Unbekümmertheit [securitas] der ungekünstelten 
Rede [oratio inadfectata]» passend - anstelle eines «auf Staunen berechnete[n]» Redestils (Quint. inst. XI 1, 93). Die ungezwungene, nicht auf Effekt ausgerichtete Schlichtheit des Stils - bei den Griechen als Aphéleia bezeichnet - könne sogar als eine Art dezenten Schmucks (ornatus purus) erscheinen, «wie man ihn auch bei Frauen liebt» (Quint. inst. VIII 3, 87). Mit einer «unberührte[n] Jungfrau» vergleicht Cicero in noch pointierterer Weise den schlichten, insbesondere der Philosophie zugeschriebenen Stil, der nicht «für den populären Geschmack geschmückt und auch nicht rhythmisiert» sei, sondern rein und zurückhaltend daherkomme - weshalb er diesen Stil auch in die Nähe der alltäglichen Unterredung (sermo) rückt und von der Rede (oratio) abgrenzt (Cic. orat. 19, 64).

Aphéleia charakterisiert ursprünglich die einfache und unverfälschte Geistesart von Kindern oder der ländlichen Bevölkerung (vgl. Bernecker 1992: Sp. 769). Damit verweist der schlichte, kunstlose, figurenarme Stil zurück auf jene ungekünstelte und unprätentiöse Art, wie sie im Grunde nur ungebildete Redner (auch Frauen, wie etwa die weiter oben genannte Laelia) aufweisen können und die gerade aufgrund ihrer einfachen Herkunft mit Ehrlichkeit und Anstand verbunden wird: «Ebenso wie durch die reiche Entfaltung von rednerischen Kunstmitteln vermag die Rede auch durch eine kunstlose Unvermitteltheit Wirkung zu erzielen, da diese den Eindruck aufrichtigen Bemühens und moralischer Integrität entstehen läßt» (Bernecker 1992: Sp. 769). Die Schlichtheit und Zurückhaltung des Stils deutet also auf die Reinheit des Charakters hin, auf eine unverdorbene und aufrichtige Rednerpersönlichkeit, die das Vertrauen und die Sympathien des Publikums verdient. Vertrauenswürdigkeit ist, wie wir wissen, ein besonders wertvolles Gut des Ethos - was die Schlichtheit des Stils auch für gebildete Redner erstrebenswert werden lässt. Doch lässt sich der implizite Widerspruch der Dissimulatio Artis hier am allerwenigsten auflösen: Unverfälschte Einfachheit lässt sich durch Kunst nicht produzieren, sondern allenfalls künstlich simulieren. Bereits der Versuch, etwas zu bewirken, ja im Grunde schon das rhetorische Bewusstsein über die Wirksamkeit des eigenen Tuns, lässt die urtümliche Schlichtheit verschwinden. Wahre Simplizität ist für die Rhetorik unerreichbar.

\subsection{Der Beifall der Menge: Natürliches Gehör und Geschmack des ungeschulten Publikums}

Betrachtet man rhetorische Situationen umfassend, darf nicht vergessen werden, dass viele Reden an ein wenig gebildetes Publikum gerichtet werden - was schon in der Antike der Fall war. Dem Aptum zufolge soll jede Rede auf ihr Publikum zugeschnitten sein, so dass sie laut Quintilian mal gewichtig und energisch, mal schmeichelnd und sanft, mal knapp und witzig sein müsse - «nicht überall das 
Gleiche, aber überall sich selbst gleichwertig» (Quint. inst. XII 10, 71). Zur Erfüllung des jeweiligen Redeziels muss der Redner zunächst die Anerkennung seines Publikums gewinnen, «und zwar nicht nur die der Kenner [doctores], sondern auch die der Menge [vulgus]» (Quint. inst. XII 10, 72). Doch wie kann der Beifall der Menge erlangt werden? Sollten die strengen Ansprüche der Rhetorik an Mäßigung, Harmonie und Perfektion gelockert werden? Welcher Grad der Anpassung an den tieferen Bildungsstand der Zuhörerschaft ist erlaubt oder gar geboten? Wenn es darum geht, die Anerkennung der Volksmenge zu gewinnen, scheinen aus Sicht der klassischen Rhetorik neben der rationalen Argumentation die Affektwirkungen sowie das Charisma des Redners zentraler zu werden. Wenn das Publikum nicht gebildet ist, hält es Cicero für besonders wichtig, dass der Redner eine Nähe zum Publikum schafft und einen überheblichen Eindruck vermeidet: Wenn der Redner «als Weiser zwischen Toren» erscheine, so hinterlasse dies bei der Zuhörerschaft entweder einen unangemessenen (ineptus) und lehrmeisterlichen Eindruck, oder der Redner wecke beim Publikum zwar Bewunderung über seine geistigen Fähigkeiten, sorge dabei jedoch für Unmut, weil die Zuhörer selbst dumm dastünden (Cic. de orat. I 221). Ein allzu gebildeter Eindruck des Redners kann hier kontraproduktiv sein. In «De Oratore` wird von Antonius entsprechend die Meinung vertreten, «seine Rede werde das Volk [populus] um so eher überzeugen, wenn man von ihm glaube, er habe überhaupt niemals studiert». Crassus behauptet gar, er versuche das Volk jeweils zu beeindrucken, indem er so tue, als würde er das Studium insgesamt verachten (Cic. de orat. II, 4).

Nicht nur der Anschein von Gelehrsamkeit, sondern auch die perfekte Anwendung der Redekunst kann angesichts eines ungebildeten Publikums unangemessen sein. Um den Geschmack der Volksmenge zu treffen, sollte gemäß Quintilian eine Rede manchmal Vorzüge aufweisen, «die als Fehler erscheinen [...]. Denn daß den Ungeschulten manchmal gefällt, was fehlerhaft ist, weiß ich wohl» (Quint. inst. XII 10, 51). Wenn man zum Volk spreche, so Quintilian, lasse sich der «Rat von Weisen» nicht immer beachten, denen zufolge weder die Leidenschaften angeregt noch für schmeichelnde Unterhaltung gesorgt werden dürfe. Ja, manchmal gelte es schlicht und einfach, die breite Masse anzusprechen, welche auch aus Ungebildeten oder Leuten vom Land bestehe, und somit «alles einzusetzen, wovon wir glauben, einen Gewinn erzielen zu können für das, was wir erreichen wollen» (Quint. inst. XII 10, 52-53). Die Anpassung an den Massengeschmack, mehr «Schwung» und «etwas hemmungsloser dargebotene[ ] Genüsse fürs Ohr» sind also durchaus geboten, wenn eine Rede ans breite Volk gerichtet wird, um «die Gemüter der ungebildeten Zuhörer» anzuregen und zu lenken (Quint. inst. XII 10, 50).

Beachtenswert ist, dass Cicero dem gemeinen Volk (vulgus) durchaus ein natürliches Empfinden (naturalis sensus) zugesteht, das darüber zu urteilen 
vermag, ob ein Redner beim Sprechen «die rechten Worte findet». Analog zu den natürlichen Sprechfähigkeiten der Laienredner bringen Cicero zufolge auch ungebildete Zuhörer bereits eine gewisse Urteilsfähigkeit mit. Insbesondere für wohlklingende und rhythmisierte Worte seien alle Menschen von Natur aus empfänglich (Cic. de orat. III 150-151) und das Laienpublikum könne problemlos zwischen richtigen und verkehrten Tönen und Rhythmen unterscheiden, selbst wenn es von der Harmonie- und Rhythmuslehre keinerlei Ahnung habe. So komme es denn auch, dass, «wenn in diesem Punkt nur ein klein wenig gesündigt wird», das ganze Publikum lauthals protestieren würde (Cic. de orat. III 195-196). Ob eine Rede ausdrucksstark und wirkungsvoll oder aber abgegriffen klingt und welche Worte gewählt und klangvoll ertönen, kann für Cicero nicht theoretisch entschieden werden, sondern bemisst sich am guten Sprachgebrauch (consuetudo bene loquendi), über den wiederum alle Menschen der Sprachgemeinschaft mit ihrem natürlichen Sinn bestimmen (Cic. de orat. III 150-151). Dabei hält Cicero alle Aspekte des Vortrags - die Stimme, die Gebärden, das Mienenspiel und insbesondere den Blick des Redners - für Bestandteile eines allgemein verfügbaren kommunikativen Repertoires, dem eine besondere vor allem affektive «naturgegebene Kraft» innewohne: «Deshalb werden auch durch sie die Laien, die breite Masse, ja selbst die Barbaren zutiefst beeindruckt» (Cic. de orat. III 223). Letztlich ist also selbst der eloquenteste und gebildetste Redner nicht über die im Alltag geltende Kommunikationspraxis und den Massengeschmack erhaben ja er darf nicht einmal automatisch davon ausgehen, besser Bescheid zu wissen als der Vulgus. Hier zeigt sich erneut Ciceros Kritik an der Schulrhetorik, seine obrigkeitskritische, fast schon demokratische Sicht, sein Aufruf zum Selberdenken, der wiederum auf seinem idealen Menschenbild bzw. Konzept der Natura aufbaut. $^{2}$

\subsubsection{Mit Blümchen glänzen, die gleich abfallen: Das Dekorative als Erfolgsfaktor oder Fehler?}

Wie weit soll sich eine Rede dem Bedürfnis des Massenpublikums nach Schmuck und Páthos anpassen? Ambivalent fällt in dieser Frage einmal mehr Quintilians

\footnotetext{
2 Gleichzeitig birgt Ciceros Anleitung zu einem natürlichen Auftritt und zur Anpassung an den Massengeschmack auch die Gefahr der Manipulation, wie Karl-Heinz Göttert kritisch bemerkt: «Die Überwältigung des Hörers ist auch noch gegen ihre Identifizierung immun - der ideale Redner als solcher unerkennbar [...]. Ciceros Rhetorik, für die Lenkung der Massen durch eine ebenso gebildete wie moralisch gefestigte Persönlichkeit gedacht, ist auch die gefährlichste Rhetorik gewesen, die je formuliert wurde» (Göttert 1994: 120).
} 
Urteil aus, wenn er sich über Reden auslässt, die besonders üppig bis schwülstig ausgestattet sind, um damit beim einfachen Volk zu punkten. Grundsätzlich hält er jede Redensart für fehlerhaft und verdorben,

die entweder sich ergeht in ungeregeltem Wortschwall oder ihr ausgelassenes Spiel mit kindischen Gedankensplittern treibt, in maßlosem Schwulst sich aufbläht oder in hohlen Gemeinplätzen schwelgt, mit Blümchen glänzt, die gleich abfallen oder unter dem Anschein des Freimutes sich in ihrem Wahnsinn austobt. (Quint. inst. XII 10, 73)

Quintilian stellt es gar als Irrtum dar, zu glauben, solcherlei Redeweisen würden beim Volk am meisten Beifall ernten. Doch gleich nach diesem abschätzigen Urteil räumt er ein, dass ein dekorativ aufgeblähter und affektiv aufgeladener Stil sicherlich vielen gefalle - und dass ihn das nicht einmal erstaune (Quint. inst. XII 10, 73). Durch eine geschickte Argumentation gelingt es Quintilian ein weiteres Mal, das Problem so zu wenden, dass Übertreibung und Fehlerhaftigkeit den Idealen des Gemäßigten und Geschliffenen am Ende unterliegen: Im Vergleich mit einer Rede, die den Redeschmuck subtil und gekonnt einsetze, würden solch vermeintliche Redeleistungen rasch verblassen, ja sogar hässlich erscheinen: «Solche Künste entfalten ihre Leuchtkraft also nur, wo das Licht der Sonne fehlt» (Quint. inst. XII 10, 76). Mit einem wuchernden, schwülstigen, verderbten Stil ließen sich zwar viele begeistern, nur mit einem guten und gemäßigten Stil könne man aber alle erreichen (Quint. inst. XII 10, 76). Es ist ein Sieg nach Punkten, weshalb tendenziell gilt: Lieber gut reden als aufgeblasen schwatzen.

\subsection{Fehler als Anzeichen von Dilettantismus}

Im Einzelnen können alle Fehler, die in einem Alltagsgespräch oder in einer Amateurrede auftauchen, auch einem ausgebildeten und geübten Redner unterlaufen - oder sie werden von diesem gerade als Kunstgriff eingesetzt. Dennoch gibt es Fehlgriffe, die als typische Laienfehler gelten können, da sie auf eine generelle Unfähigkeit oder Unbeholfenheit, auf mangelhafte Sprachbeherrschung oder zumindest auf wenig Fingerspitzengefühl hindeuten und somit zum Erkennungsmerkmal des ungeschulten Redners werden.

\subsubsection{Barbarismus und Vulgarität}

Zwei Formen des Fehlers sind schon von der Bezeichnung her an die Rede der ungebildeten, breiten Masse gekoppelt: der Barbarismus und das Vulgäre. Den 
Barbarismus haben wir bereits als grammatischen Fehler in den Einzelwörtern kennengelernt (siehe Kap. 2.4.1). Geht man vom Wortstamm des Barbarismós aus, sind Barbarismen eigentlich als Fehler der Barbaren, d.h. als Redeweisen der unzivilisierten, rohen, ungebildeten Menschen und somit als grundlegende Ausdrucksformen des dilettantischen Sprechens zu interpretieren. In der Antike bezog sich Bárbaros ursprünglich auf die fehlerhafte Rede einer stammelnden (ঝbar-bar `-sagenden) Person und stand als Überbegriff generell für alle fremden bzw. nichtgriechischen Menschen. Bis heute wird mit «Barbar» auch ein besonders ungehobelter Mensch bezeichnet. In der klassischen Rhetorik spiegeln sich alle drei Bedeutungsrichtungen: Erstens benennt der Barbarismus, wie gesagt, einen Fehler in der Schreibweise oder Aussprache eines Wortes, zweitens das unzulässige Einbauen von Fremdwörtern in die Rede und drittens generell eine Geistesverfassung, die mit einer gewaltsamen und ungezügelten Sprechweise einhergeht (Quint. inst. I 5, 8-10). Barbarismen sind damit zwar die deutlichsten Anzeichen von Dilettantismus aufgrund mangelnder Sprachbeherrschung, doch können selbst offensichtliche Laienfehler strategisch in der Rede eingesetzt werden: Die Abänderung von Wortbestandteilen ebenso wie die Verwendung umgangssprachlicher oder sprachfremder Wörter können als Figuren der Rede eingesetzt werden und so zum Ausdruck der Redekunst werden (vgl. 2.2 und 2.4). Beide Prozesse sind überdies Bestandteil der natürlichen Veränderung und Entwicklung jeder Standardsprache. ${ }^{3}$ Selbst Rohheit und ungezügelt zur Schau gestellte Gefühle in der Rede können zur Verstärkung der Emotionalität und Authentizität der Rede bewusst eingesetzt werden (vgl. 2.5.1). Nicht immer ist klar, ob es sich um einen «echten〉 Barbarismus oder ein gezielt eingesetztes Kunstmittel handelt.

Als zweites Merkmal der Sprechweise des gemeinen Volks (vulgus) lässt sich der Fehler der Vulgarität (vulgaris) bezeichnen. Das Vulgäre wird in der klassischen Rhetorik mit dem Obszönen, dem Schmutzigen, dem Hässlichen oder ganz einfach mit dem Niedrigen und Gewöhnlichen in Verbindung gebracht (Quint. inst. VIII 2, 1; X 1, 9). Niedrig (humilis) oder gar unanständig (sordidus) wirke etwa die Metapher «Felsenwarze», hässlich (deformis) die Rede von der «Kastration» des Gemeinwesens (Quint. inst. VIII 6, 14f). Während obszöne, d.h. gegen die Sitt-

3 Die in den klassischen Texten zu findenden Beispiele von Barbarismen werden aus heutiger Sicht als «wertvolle Zeugnisse des Vulgärlateinischen und der Anfänge der romanischen Sprachen» betrachtet (Erlebach 1992b: Sp. 1281). Von den Lateinern noch als Fehler betrachtet, weisen sie bereits auf eine Weiterentwicklung des Lateinischen hin. Im Purismus des 17. und 18. Jahrhunderts gab es den Versuch, mittels Lehrbücher, «Antibarbarus` genannt, solchen Tendenzen der Sprachentwicklung (oder -verunreinigung) entgegenzuwirken. Sie richteten sich gegen jede Abweichung von der Sprachreinheit, insbesondere gegen die barocke Manieriertheit (vgl. Erlebach 1992b: Sp. 686-688). 
samkeit verstoßende Wörter (verba parum verecunda) durch den Redner in jedem Fall zu meiden seien, könnten die übrigen laut Quintilian jedoch in einer Rede durchaus ihren Platz haben, «denn auch niedrige [humilis] und sogar gemeine [vulgaris] Wörter sind zuweilen angebracht, und was an einer glänzenderen Stelle sich häßlich ausnimmt, ist da, wo es die Sache erfordert, der eigentliche Ausdruck» (Quint. inst. X 1, 9).

Auch humoristisch gemeinte Zweideutigkeiten können als Merkmale des Vulgären gelten. Solche könnten Quintilian zufolge zwar auch in der Rede ausgebildeter Redner ihren Platz haben, um das Publikum zum Lachen zu bringen, doch dürften sie keinesfalls obszön (amphibolia obscaena) oder allzu primitiv ausgestaltet werden, d.h. von der Art, «wie sie im Volke gerade die gewöhnlichsten Menschen [vulgus vilissimus] im Munde führen» (Quint. inst. VI 3, 47). Unanständig (deformis) aufgrund ihrer Zweideutigkeit wäre etwa die Wortkombination «cum notis» (mit Bekannten), weil dies klanglich mit «cunnotis», der weiblichen Scham, assoziiert werden könnte. Solcherlei 〈Vulgaritäten〉 kennzeichnen aus Sicht der klassischen Rhetorik also die Laienrede bzw. die Rede des ungebildeten Volks oder Vulgus. Als gebildeter Redner müsse man auf der Hut sein, dass der eigenen Rede kein unanständiger Sinn untergeschoben werde (Quint. inst. VIII 3, 47). Allerdings ließen sich mit bösem Willen selbst Ausdrücke, «die von Obszönität [obscenitas] meilenweit entfernt sind», noch auf hässliche Weise ausdeuten: «Wenn man dies bedenkt, gibt es überhaupt nichts, was man unbedenklich sagen könnte» (Quint. inst. VIII 3, 47). Vulgarität kann also auch erst durch die Interpretation entstehen - über die der Redner selbst jedoch nur beschränkt Kontrolle hat.

\subsubsection{Inornatus: Vom blutleeren Stil bis zum Kitsch}

Wie bei allen Tugenden der Rede gibt es auch in Bezug auf den Ornatus sowohl den Fehler des Zuwenig als auch den Fehler des Zuviel: «Indessen ist es nun freilich mit der schönen Wirkung des Schmucks so, daß die Rede ohne Zusätze nackt (nudus) und gleichsam ungepflegt (incomptus), mit einer Vielzahl von Zusätzen jedoch überladen erscheint» (Quint. inst. VIII 6, 41). Für die Laienrede können nun gleich beide Extreme bzw. Ornatus-Fehler charakteristisch sein. So kann die Rede Ungebildeter entweder durch Schmucklosigkeit auffallen, weil sie unzureichend mit Figuren ausgestattet (aschēmátiston) ist, oder im Gegenteil gerade weil sie in übertriebener Weise ausgeschmückt - mit Blümchen ausgestattet - ist (kakózēlon) (Quint. inst. VIII 3, 59; 56). Fällt die Redeweise allzu einfältig aus, kann die natürliche Tugend der Ungebildeten, die sich ja in ungekünstelter Schlichtheit oder Aphéleia ausdrückt, dem Auctor ad Herennium zufolge 
auch zum Vitium des trockenen und blutarmen Stils (aridum et ex[s]angue genus orationis) geraten (Rhet. Her. IV 11, 16). Quintilian gilt insbesondere der Fehler der Gleichförmigkeit (homéideia) in Satzbau, Figurenanwendung und Compositio als Zeichen dafür, dass es einem Redner an Kunstverstand fehlt (carens arte) (Quint. inst. VIII 3, 52). Wenn alles aus einer Färbung besteht und jeglicher Reiz der Abwechslung fehlt, kann nur ein Anfänger am Werk sein.

Umgekehrt können gewisse Formen des Überschwänglichen und Schwülstigen und ein gewisser Hang zum Gesuchten ebenfalls typisch für ungebildete Redner sein (Quint. inst. VIII 3, 56). Unpassende oder ungereimte Metaphern, übersteigerte Hyperbeln oder verpfuschte Allegorien kommen ebenfalls bei Laien vor (Quint. inst. VIII 6, 50-52). Anders als beim Übereifer, der gebildete Redner zur Übertreibung treibt, greifen Laienredner vielmehr aufgrund mangelnder sprachlicher Gewandtheit und Geschmacksbildung zu übertriebenen oder inadäquaten Schmuckmitteln. Diese antike Form des «Kitschs〉 lässt sich durch einen Verstoß gegen das Aptum erklären, der auf einer «Absolutsetzung des delectare» beruht (Kalivoda 1998: Sp. 950). Indem der ungebildete Redner den Genuss, die «Gefühlspotenzierung» (950) seiner Rede über alles andere stellt, ergibt sich bei ihm eine Präferenz für das opulent Ausgeschmückte oder - wie wir heute sagen würden - für das Kitschige.

\subsubsection{Ohne Form und Inhalt: Unförmiger Ausdruck, leeres Gerede}

Die Aposiopese, d.h. den abrupten Abbruch eines Satzes oder Gedankens, und das Asyndeton, d.h. die unverbundene Rede, haben wir als Strategien der Imperfektion in der Rede kennengelernt, die dazu dienen, der eigenen Emotionalität Ausdruck zu verleihen oder dem Publikum das Gesagte gleichsam «einzuhämmern` (vgl. Kap. 2, 2.2.2). Ebenso gut kann jedoch die abrupte oder abgerissene Art $\mathrm{zu}$ reden ungewollt entstehen, wobei dies als «Indiz einer mangelnden Ausdrucks- und Gedankenbeherrschung» (Plett 1971: 60) und somit als Dilettantismus zu deuten wäre. Quintilian ordnet die zerhackte oder abreißende Rede, wie wir weiter oben gesehen haben, denn auch explizit der Laienrede zu (Quint. inst. II 11, 6-7). Neben dem abgehackten Stil oder dem unverbundenen Aneinanderreihen von Wörtern können auch andere Elaborationsmuster der Rede, die einen inkonsequenten, holprigen, unförmigen, arhythmischen oder missklingenden Redefluss nach sich ziehen, als eigentliche Laienfehler identifiziert werden (siehe z.B. Quint. inst. IX 4, 56).

Doch nicht nur die defizitäre Form der Rede, sondern auch ein Mangel an inhaltlicher Aussagekraft kann aus der Unkenntnis der rhetorischen Grundregeln oder der Ungeübtheit im Sprechen resultieren. Besonders bei Quintilian finden 
wir eine klare Zurückweisung jeder Form des «nichtssagenden Worterguss[es]», des unüberlegten, zufälligen Redeschwalls oder des zusammenhangslosen «Wortgetümmel[s]», die er sämtlich auf einen Mangel an rhetorischer Schulung (und an Bildung überhaupt) zurückführt (Quint. inst. X 7, 12-13; 22). Cicero sieht die Ursache inhaltsleeren Geredes ebenfalls in der fehlenden Allgemeinbildung und Sachkenntnis des Redners, «denn aus dem Wissen um die Sache muß die Rede in Glanz und Fülle des Ausdrucks erwachsen. Hat sich der Redner die Sache nicht ganz angeeignet, so bietet seine Rede nur leeres [inanis] und beinahe kindisches [puerilis] Geschwätz» (Cic. de orat. I, 20). Das «Gewirr leerer Worte» (turba inanium verborum), von dem auch Quintilian spricht und das sich durch eine wortreiche und doch nichtssagende Geschwätzigkeit auszeichnet, zeugt von Dilettantismus, der seinen Ursprung im Übereifer des unzureichend gebildeten Redners hat (Quint. inst. VIII 2, 17).

\subsubsection{Mixtura verborum: Stilbruch, sprachliche Vermischung und Stilmix}

Wenn Stil und Sprache nicht dem Redezweck oder den Regeln des Anstands angepasst sind und somit das Aptum bzw. Decorum nicht einhalten, deutet dies in der klassischen Rhetorik auf dilettantisches Sprechen hin. Stilistische Verstöße gegen das Decorum sind innerhalb des klassischen rhetorischen Lehrsystems nicht vorgesehen und werden auch nicht als Strategie der Imperfektion empfohlen. Die Angemessenheit scheint somit jene Tugend der Elocutio zu sein, die unter keinen Umständen umgangen werden darf, während im Sinne der Angemessenheit eine Abweichung bzw. Relativierung der anderen Tugenden, also der Klarheit, des Schmucks oder der korrekten Sprache, möglich ist.

Quintilian nennt als Anzeichen dafür, dass ein Redner die Klaviatur der Stile und Stilhöhen nicht beherrscht, den Fehler, «Erhabenes mit Niedrigem, Altes mit Neuem, Poetisches mit Gewöhnlichem [vulgaribus] zu vermischen» (Quint. inst. VIII 3, 60). Die Vermengung von Wörtern, die eigentlich unterschiedlichen Stilhöhen zuzuordnen wären, wird auch als Fehler des Coenismus (koinismós) bezeichnet (vgl. Joseph 1994: Sp. 262-263). Werden in der Rede gar Merkmale unterschiedlicher Sprachen oder Dialekte zusammengemischt, z.B. «wenn man unter Attisches Dorisches, Ionisches und Äolisches mischt» (Quint. inst. VIII 3, 95), ergibt sich in der Rede eine Art «Kauderwelsch», ein Sprachenmix (quedam mixta ex varia ratione linguarum oratio), den Quintilian als Sardismós bezeichnet (Quint. inst. VIII 3, 95). Den Fehler des Sardismós vergleicht Quintilian nach Horaz mit einem heterogen aufgebauten Bild: «Wenn ein Maler dem menschlichen Haupt einen Pferdehals geben will ... und auch die anderen Glieder von Wesen verschiedener Art anfügte» (Quint. inst. VIII 3, 60). Die «Wortvermi- 
schung» (mixtura verborum) kann laut Quintilian den Fehler der Dunkelheit (obscuritas) hervorrufen (Quint. inst. VIII 2, 14). Mangelndes Sprachgefühl, fehlende Übung, mangelhafte Bildung - verschiedene Ausprägungen des Dilettantismus also - können diese Formen der Sprachvermischung oder des Stilbruchs hervorbringen. Aristoteles zufolge wirkt die Vermengung von Ausdrücken unterschiedlicher Stilhöhen komisch oder grotesk, weshalb sich dieses in der Rede unangemessene Stilmittel entsprechend wirkungsvoll in der Komödie einsetzen lasse: «O du erhabener Feigenbaum!» (Aristot. rhet. III 7, 2, 1408a-b).

Für die Rhetorik selbst wird der Stilbruch als Figur oder gezieltes Wirkungsmittel allerdings in der Antike noch nicht entdeckt: Er ist aus Sicht der klassischen Rhetorik stets als Vitium zu deuten (vgl. Scheichl 2012: Sp. 1257). ${ }^{4}$ Inzwischen wird das rhetorische Potenzial des Stilbruchs in der Rede und in anderen Künsten aktiv genutzt, doch immer noch stehen den Beispielen des kunstvollen Stilbruchs «im Alltag zahlreiche Fälle schlicht und einfach mißlungener Formulierungen gegenüber, die auf einem Stilbruch beruhen» (Scheichl 2012: Sp. 1278).

\subsection{Fazit}

Wie also äußert sich die klassische Rhetorik zur «Rhetorik des Amateurs»? Als Kunst, die nach Perfektion strebt und entsprechende Sorgfalt, Bemühung, Bildung und Einübung einfordert, distanziert sie sich grundsätzlich von allen dilettantischen Elaborationsformen der Rede. Dennoch konnte in dieser Frage eine unerwartet ambivalente Haltung aufgezeigt werden.

Die Alltagsrede bzw. die normale Umgangssprache steht für Natürlichkeit. In ihr steckt die Kraft des Ungehobelten, sie verströmt einen ungekünstelten Reiz und sie ist nahe beim Publikum. Um diese Wirkkraft zu nutzen und den Anschein der Künstlichkeit zu vermeiden, nimmt sich die Rhetorik immer wieder die gewöhnliche Art zu reden zum Vorbild - auch wenn sie sich als Kunst gerade davon distanziert. Die Rede ist, anders als die übrigen Künste, eine Téchnē, in der grundsätzlich alle Menschen Expertise und Gewandtheit mitbringen. Hebt sich eine Rede zu stark von der normalen Umgangssprache ab, droht die Gefahr eines unnatürlichen und befremdenden Klangs. Gewisse Qualitäten der ungekünstelten Laienrede werden daher von der Rhetorik anerkannt oder sogar gesucht, gleichzeitig wird aber jede Form von Geschwätzigkeit und Derbheit verworfen.

4 Der Eintrag zum «Stilbruch〉 im «Historischen Wörterbuch der Rhetorik〉 nennt keine einzige klassische rhetorische Quelle, ja geht nicht einmal auf den Sardismós bei Quintilian oder die Komik der Stilhöhenverschiebung bei Aristoteles ein (Scheichl 2012), bezeichnet aber Lexikoneinträge zu «Sprachmischung〉 und «Stilkreuzung〉 (Sp. 1257). 
So wird auch der Orator vulgaris oder Laienredner für seine Grobheit kritisiert, findet aber zugleich Bewunderung für seinen unbeschwerten Schwung und seine Spontaneität. Die schlagfertige und gewitzte Art des Laienredners wird auch als Zeichen von Menschlichkeit gesehen. Viele Fehler, die aufgrund von Dilettantismus geschehen, sind gerade bei einem ungebildeten Publikum nicht ungern gesehen, so etwa der Hang zum Dekorativen, Gefälligen und Affektgeladenen. Da allen Menschen ein natürliches Sprachgehör attestiert wird, ist selbst der gebildetste Redner nicht zwingend über den Massengeschmack erhaben. Charakteristisch für den Redestil alltäglicher oder wenig bedeutender Situationen ist Schlichtheit und Schnörkellosigkeit sowie der Verzicht auf Pathos. In seiner unverfälschten Zurückhaltung und Reinheit kann der schlichte Stil fast wieder als Schmuck erscheinen - und Rückschlüsse auf die moralische Integrität und Aufrichtigkeit des Redners nahelegen. Den Anschein des Unprätentiösen und Integren, den der schlichte, unverformte Redestil einfacher Leute auf «natürlichem` Weg mit sich bringt, würde sich die klassische Rhetorik gerne zu eigen machen, um Vertrauen zu schaffen. Dabei tritt jenes Paradoxon besonders virulent zu Tage, das die Dissimulatio Artis insgesamt kennzeichnet: «Es ist die Schlichtheit als die einer Verstellung eigentlich unzugängliche Simplizität, die das Täuschen unwahrscheinlich macht» (Danneberg 2006: 60).

Die Merkmale der Laienrede sind aus Sicht der klassischen Rhetorik eigentlich als Fehler zu bewerten, die aus der mangelhaften Beherrschung der Redekunst, d.h. aus Dilettantismus erfolgen. Dennoch ist in den antiken rhetorischen Schriften eine Ambivalenz gegenüber der «Rhetorik des Amateurs` angedeutet: Die Rede rhetorisch Ungebildeter kann eine Reihe positiver Wirkungsweisen entfalten, die sie der elaborierten, perfektionierten Rede voraushat. Die genannten Fehler und Vorzüge der Laienrede werden hier noch einmal im Überblick präsentiert. 


\section{Die Fehler und Dilettantismen der Amateurrede}

- Barbarismus: ungebildet, unüberlegt, stammelnd, grammatikalisch fehlerhaft, falsch ausgesprochen, fremd, provinziell, grobschlächtig, roh, ungehobelt, zügellos, grob, gewalttätig

- Vulgarität: obszön, unanständig, schmutzig, hässlich, niedrig, banal, gewöhnlich

- Schmucklosigkeit (aschēmátiston): trocken, kahl, blutleer, ungepflegt, plump, einfältig

- Gleichförmigkeit (homeídeia): langweilig, reizlos, einfältig, ohne Abwechslung

- Überfrachtung (kakózēlon): überladen, schwülstig, übersteigert, überflüssig, gesucht, abgeschmackt, süßlich, kitschig, effekthascherisch

- Formlosigkeit (anoikonómēton) und fehlender Zusammenhang (kakosýntheton): abgerissen, gebrochen, zerhackt, holprig, ungefügt, ungeordnet, arhythmisch, missklingend, regel- und pausenlos sprudelnd, zusammengestückelt; fehlende Ganzheit

- Inhaltslosigkeit: nichtssagend, leer, geschwätzig

- Stilbruch (sardismós): Vermischung unterschiedlicher Stilhöhen, Stilmix

\section{Die Vorzüge der Amateurrede}

- Natürlichkeit: ungekünstelt, unverstellt, ungeschliffen, naturgemäß, urwüchsig, ungeniert, ungezwungen, ungehemmt, gelassen, frei

- Vertrautheit: üblich, gewöhnlich, gewohnt, vertraut, verständlich, normal, alläglich, nahbar

- Gewandtheit: sicher, selbstverständlich

- Spontaneität: unmittelbar, spontan, leicht, schlagfertig, gewitzt

- Schlichtheit: rein, bescheiden, zurückhaltend, unprätentiös, dezent, unauffällig, subtil

- Integrität: aufrichtig, ehrlich, menschlich, glaubwürdig, arglos

- Authentizität: echt, unverfälscht, wahrhaftig

- affektive Kraft: wuchtig, schwungvoll, leidenschaftlich, gefühlvoll, wortmächtig, energisch, eindringlich, berührend 